\title{
DIEZ RAZONES PARA ORGANIZAR SU IGLESIA EN GRUPOS PEQUEÑOS
}

\author{
Rubén Dagoberto Montero Guerrero \\ Universidad Peruana Unión \\ rubenmontero@teologia.edu.pe
}

Si hay muchos miembros de la iglesia, organícense en pequeños grupos para trabajar no solo por los miembros

de la iglesia, sino en favor de los incrédulos. Si en algún lugar hay solamente dos o tres que conocen la verdad, organicense en un grupo misionero. Mantengan intimo su vínculo de unión, cerrando sus filas por el amor y la unidad, estimulándose unos a otros para progresar y adquiriendo cada uno valor, fortaleza y ayuda de los demás

Elena G. de White, 1902

Fecha de recepción: Noviembre 2014. Fecha de aceptación y versión final: Diciembre 2014.

El autor enumera diez razones por las que es vital la implementación del plan de grupos pequeños en la iglesia. Si bien, este artículo está dirigido principalmente a aquellos lideres que todavía no se han posicionado firmemente a favor del establecimiento de grupos pequeños en su iglesia, también será útil para aquellos líderes que empezaron la implantación de los grupos pequeños, pero que por alguna razón están pasando por dudas o frustraciones al ver pocos resultados positivos en sus grupos pequeños.

Palabras clave: Iglesia, grupos pequeños, liderazgo, evangelismo. 


\section{TEN REASONS TO ORGANIZE YOUR CHURCH IN SMALL GROUPS}

If there is a large number in the church, let the members be formed into small companies, to work not only for the church members, but for unbelievers. If in one place there are only two or three who know the truth, let them form themselves into a band of workers. Let them keep their bond of union unbroken, pressing together in love and unity, encouraging one another to advance, each gaining courage and strength from the assistance of the others.

Ellen G. White, 1902.

\section{Abstract}

This article lists ten reasons why implementing small groups in the Church is vital. This article is aimed mainly at those leaders who still do not have positioned themselves firmly in favor of establishing small groups in their Church. It will also be useful for leaders who began the implementation of small groups, but which for some reason are going through doubt or frustration because they experience poor results.

Keywords: Church, Small Groups, leadership, evangelism 


\section{Introducción}

Los grupos pequeños existen simplemente porque trabajar en grupos pequeños es la manera natural y espontánea en que trabajamos los seres humanos. Durante nuestra vida siempre estamos en contacto con grupos pequeños: la familia, los amigos en la escuela, los amigos de la juventud, los grupos de trabajo, los directorios de las empresas, las juntas de iglesia, etc. Todos pertenecemos de alguna manera u otra a 44 algún grupo pequeño.

Sin duda, hay razones poderosas que los seres humanos tenemos para constituir grupos y alcanzar los más diversos objetivos; entre ellas está el hecho que trabajando en grupo logramos hacer mejor muchas cosas que no podríamos hacer solos. Una característica distintiva de las organizaciones de mayor éxito es que han construido una atmósfera y una cultura interna en que el trabajo grupal alcanza un nivel de eficiencia mayor que el que alcanzarían sus miembros si trabajasen por separado.

El gran paso se da cuando los grupos pequeños pasan de ser espontáneos e inorgánicos a ser planificados y con una estructura funcional que le permita alcanzar sus objetivos con mayor eficacia. Ese gran paso es el que ha decidido dar nuestra iglesia al enfatizar la formación de grupos pequeños en todas las congregaciones adventistas. Ahora bien, organizar la iglesia en grupos pequeños puede ser considerado como una verdadera revolución. Porque se trata básicamente de salir de un modelo de adoración y evangelismo centrado en programas en el templo hacia un sistema más dinámico, sobre la base de los grupos pequeños en las casas. En este nuevo paradigma, los eventos eclesiásticos apuntan al fortalecimiento y la consolidación del crecimiento espiritual de la iglesia a través de un sistema de grupos pequeños bien organizados.

En la última década, nuestra iglesia ha colocado acer- 
tadamente como una de sus principales prioridades el establecimiento de grupos pequeños. Sin embargo, a pesar de las innumerables arengas que se han dado y de las múltiples capacitaciones que se han promovido en los últimos años, todavía existen significativos sectores de la iglesia que mantienen una cierta resistencia a la implantación decidida de los grupos pequeños. Para algunos líderes, la organización de los grupos pequeños en su iglesia se ha convertido en una carga pesada, un oneroso fardo que sobrellevan con cierta dosis de resignación. Lo cierto es que ninguna iglesia llegará lejos en la organización del plan de grupos pequeños sin el liderazgo y el comprometimiento activo de sus líderes más influyentes, especialmente los pastores y ancianos. En ese sentido, este artículo tiene el propósito de enfatizar la importancia y la urgencia de implantar los grupos pequeños en cada una de nuestras iglesias.

\section{La historia de María}

El ministerio de los grupos pequeños provee la oportunidad de ver los milagros que Dios obra en el corazón de las personas. El caso que se presenta a continuación, es apenas una muestra de lo que ocurre continuamente en el contexto del plan de grupos pequeños.

María tenía grandes problemas personales y familiares. El esposo la había abandonado después de más de veinte años de matrimonio, corriendo atrás de una mujer mucho más joven. Uno de sus hijos había entrado en el mundo de las drogas y cada vez se hundía más en el horrible círculo destructivo que lo estaba convirtiendo en un muerto viviente. A causa de los problemas que enfrentaba, María cayó en una terrible depresión que la hacía no querer seguir viviendo más. Fue acariciando la idea del suicidio, hasta que un día decidió acabar de una vez con todo ese sufrimiento quitándose la vida. María tomó algunas pastillas y quedó 
en estado de inconciencia hasta que despertó en la cama de un hospital. Mientras estaba convaleciente, una de sus amigas más queridas, Cecilia, que era miembro de la Iglesia adventista, la había visitado todos los días. Cecilia le habló acerca de su grupo pequeño, y le contó cuánto bien le habían hecho esas reuniones. María disfrutaba de la compañía de Cecilia, así que se pusieron de acuerdo para asistir juntas a la reunión.

El día de la reunión del grupo pequeño, Cecilia llegó pun46 tualmente. Minutos antes, María había estado pensando que quizá no era una buena idea asistir al grupo pequeño. Le pareció que no se sentiría bien entre gente extraña y pensó en inventar alguna excusa para no asistir. Pero al ver a su amiga Cecilia, que la había visitado en el hospital y que había hecho tanto por ella, se dijo que haría un pequeño sacrificio y la acompañaría a la reunión.

A María le agradó la amabilidad con la que fue recibida en la reunión del grupo peque- ño. Sin embargo, la carga emocional que sentía por sus problemas personales hizo que se retrajera, mientras otros invitados conversaban animadamente. Pronto se dio cuenta que había llegado el momento de los pedidos de oración y pensó que podía pedir que oren por ella, por los problemas que estaba enfrentando. La líder del grupo pequeño le preguntó si tenía algún pedido de oración y ella pudo tener la oportunidad de hablar de sus problemas. Se dio cuenta que la líder le prestaba atención, y le hacía preguntas para conocer más de cerca la situación que ella enfrentaba. Se sorprendió a sí misma cuando empezó a contar su historia como si conociera a esas personas de toda la vida. Se sentía feliz al tener a personas dispuestas a escucharla. Mientras contaba su drama, revivía los sentimientos de angustia y el llanto era inevitable. Todos escucharon con atención hasta que María terminó. Luego, uno a uno, los miembros de ese grupo pequeño empezaron a decir- 
le palabras de ánimo. Algunos abrían la Biblia y leían preciosas promesas que María nunca había escuchado y que le parecían escritas especialmente para ella. Nadie le había dado mensajes tan hermosos. Después de haber compartido la Palabra de Dios, oraron por ella. María sintió cómo se aligeraba su carga y cuando terminó la oración, ella se puso de pie sintiéndose una nueva persona.

María regresó todos los viernes a la reunión del grupo pequeño. El mensaje de la Biblia le parecía un alimento extraordinario y María quería saber más y más de Jesús. Un día le ofrecieron estudiar la Biblia en su casa, y ella aceptó con alegría. El curso bíblico fue un poderoso elemento para consolidar sus nacientes convicciones adventistas. Tres meses después, María se bautizó. Su bautismo fue realizado en el marco de una ceremonia inspiradora. Al salir de las aguas, las primeras personas que se acercaron para abrazarla, fueron los miembros de su grupo pequeño. Fue un momento de gozo extraordinario, porque esta mujer había estado en el fondo del pozo de la angustia y la depresión y ahora se gozaba en la salvación de Cristo Jesús.

El grupo pequeño continuó apoyando a María. Continuamente la visitaban en su casa y ella nunca se perdía las reuniones. María aprendió a compartir la historia de su vida y de las grandes cosas que Dios hizo por ella. Actualmente, María es una cristiana que verdaderamente ama a Jesús y tiene un profundo compromiso misionero. Su mayor alegría es conducir a las personas a los pies de Jesús. Y, por supuesto, es líder del grupo pequeño que se reúne en su casa.

María ejemplifica cómo el trabajo del grupo pequeño unido a la obra del Espíritu Santo es una herramienta poderosa para el evangelismo y la consolidación de la experiencia cristiana. Si solo nos remitiésemos a los muchos testimonios de vidas transformadas en el ministerio de los grupos pequeños, tendríamos poderosos 
argumentos para implantarlos en cada iglesia. Sin embargo, permítame mostrarles a continuación otras diez razones por las que se hace necesario profundizar nuestra aplicación de los grupos pequeños en cada congregación adventista, grande o pequeña.

\section{Un sólido fundamento bíblico}

Como iglesia, debemos tener la seguridad que nuestras actividades y nuestros métodos de evangelismo tengan un sólido respaldo en la Palabra de Dios. Elena de White declaró: "Una frase de la Escritura tiene más valor que diez mil ideas o argumentos humanos". ${ }^{1}$ Este principio se aplica con mayor razón al ámbito de las actividades misioneras. Si bien, la Biblia no describe en detalle los métodos misioneros que debemos aplicar, sin embargo, ella sí nos

${ }^{1}$ White, Joyas de los Testimonios, 3: 110 . presenta los grandes lineamientos que hemos de adecuar a los contextos específicos en que se hace la labor evangelística.

Solo mencionaré tres ejemplos contundentes del exitoso empleo del sistema de grupos pequeños descritos en la Biblia: a) la organización del pueblo de Israel en el desierto, ${ }^{2}$ b) el

${ }^{2}$ En Éxodo 18 se describe la organización del pueblo de Dios en el desierto. El punto central en esta organización es el empleo de una estructura de liderazgo bien definida. Al seguir la intuición certera de Jetro, Moisés realizó una labor de reingeniería administrativa que le permitió liderar con mayor eficiencia al pueblo de Israel. Se edificó una pirámide de liderazgo, donde en la base se encontraban los líderes de diez, luego estaban los de cincuenta, de cien y de mil, para finalmente llegar a Moisés. En ese contexto, es evidente que la parte más difícil era la búsqueda, la selección y la preparación de esos setenta y ocho mil seiscientos líderes que le hacían falta para que el pueblo pueda ser bien administrado. Esa estructura de liderazgo era la columna vertebral que tenía Moisés para dirigir al pueblo. Hay diferencias marcadas entre los modernos grupos pequeños y la estructura administrativa de Moisés, sin embargo el principio subyacente es el mismo: la división en grupos pequeños ayuda a realizar más eficientemente la administración y el cuidado del pueblo de Dios. 
grupo pequeño de Jesús y sus discípulos, ${ }^{3}$ c) las iglesias en las casas de la iglesia primitiva. ${ }^{4}$ En

${ }^{3}$ Es significativo el hecho que Jesús llamó a un grupo reducido de sus seguidores. Según Marcos 3:14, el propósito que tuvo era "que estuviesen con él, y para enviarlos a predicar”. Del mismo modo, los grupos que se formen en la iglesia deben tener el doble propósito de estar en relación con Jesús y de predicar el evangelio, en otras palabras, comunión y misión. Por otro lado, después de establecer su círculo más íntimo de discípulos, Jesús los envió a predicar de dos en dos, mostrando que no existe incompatibilidad alguna entre el establecer grupos pequeños y el trabajo de las parejas misioneras, ambos ministerios pueden y deben formar parte de una estrategia integral de la actividad misionera de la iglesia.

${ }^{4}$ Aunque la iglesia primitiva no tenía un sistema de grupos pequeños como el conocemos en la actualidad, sí podemos inferir que las reuniones celebradas en las casas, de las que hay abundante registro bíblico, eran de grupos pequeños de cristianos. Las cartas apostólicas eran leídas en aquellos grupos pequeños de cristianos que eran las iglesias del primer siglo. (Ver Ro 16:5, 1 Co 16:19, Flm 1:2. Compárese con Mt 18:20). Se puede argumentar que estas iglesias en las casas fueron el resultado de la persecución que soportaban los cristianos, sin embargo, es un hecho indiscutible que todas las iglesias del Nuevo Testamento fueron de grupos pequeños de cada uno de estos casos, los grupos pequeños son una herramienta eficaz para alcanzar los objetivos divinos.

Es evidente que muchos de los detalles que suelen acompañar la implementación de los grupos pequeños son desconocidos en la Biblia. No hay referencias claras, por ejemplo, a la realización de desfiles de grupos pequeños, ni la organización de fórums o congresos de líderes de grupos pequeños, o a la capacitación semanal de los líderes y otros aspectos del plan de los grupos pequeños en la iglesia. Del mismo modo, como en el caso del evangelismo público, no hay referencias al maestro de ceremonias, a los sorteos, y, en general a la programación que caracteriza nuestros ciclos de conferencias

cristianos, y que el crecimiento del cristianismo fue exponencial en ese tiempo, en tanto que cuando finalmente se edificaron grandes templos, y se oficializó la religión cristiana como religión del Imperio Romano, sobrevino la apostasía y el periodo de oscurantismo religioso que predominó en el cristianismo de la Edad Media. 
y seminarios evangelísticos. Sin embargo, nadie puede dudar que los fundamentos del evangelismo público se encuentran en la Biblia, así también es innegable tanto en el Antiguo como en el Nuevo Testamento que el pueblo de Dios aplicaba el ministerio en grupos pequeños.

\section{Un sólido fundamento en el Espíritu de Profecía}

Para los adventistas es una gran bendición contar con el ministerio profético de Elena

50 G. de White. Sus consejos han sido determinantes en el éxito de los emprendimientos misioneros de la iglesia. En relación con el establecimiento de grupos pequeños, tenemos abundantes referencias en los escritos de Elena de White. Veamos las que enfatizan la necesidad de implementar los grupos pequeños. Esta primera cita nos muestra que nuestras iglesias deben organizar grupos para el servicio, lo que indica que los ganadores de almas deberían unirse formando equipos de trabajo para llevar el mensaje a quienes no conocen a Jesús:

"En nuestras iglesias deben organizarse grupos para el servicio. En la obra del Señor no ha de haber ociosos. Únanse diferentes personas en el trabajo como pescadores de hombres. Traten de recoger a las almas de la corrupción del mundo y conducirlas a la pureza salvadora del amor de Cristo". 5

Ella es mucho más enfática en esta siguiente cita, porque declara que los grupos pequeños deben ser la "base del esfuerzo cristiano". Lo que indica que no puede haber un verdadero esfuerzo misionero sin formar grupos pequeños, ya que ellos son la base. Además ella remarca que este es un plan que proviene del mismo Dios, quien "no puede equivocarse":

"La formación de pequeños grupos como base de esfuerzo

${ }^{5}$ White, Evangelismo, 88. 
cristiano, es un plan que ha sido presentado ante mí por aquel que no puede equivocarse". ${ }^{6}$

La siguiente cita nos muestra el énfasis de los grupos pequeños en el estudio de las Escrituras. Según Elena de White, no hay otra manera de mantener la integridad del cristiano, sino a través de alimentarse de la Palabra de Dios en un círculo de apoyo cristiano, que es el grupo pequeño:

"Reúnanse pequeños grupos para estudiar las Escrituras. No perderán nada y ganarán mucho. Los ángeles del cielo asistirán a sus reuniones y al alimentarse con el pan de vida recibirán fortaleza espiritual. Se estarán alimentando, por así decirlo, con las hojas del árbol de la vida. Solo así mantendrán su integridad".

Elena G. de White también presenta a los grupos pequeños como el escenario ideal para que los creyentes profundicen su amor por las almas y para que el Espíritu pueda desplegar todo su poder en favor de quienes no conocen a Jesús:

"Existe una gran necesidad de que se practique la oración secreta, pero también se necesita que varios cristianos se congreguen y se unan para presentar fervorosamente sus peticiones delante de Dios. En esos grupos pequeños Jesús está presente, se profundiza el amor por las almas en el corazón y el Espíritu despliega sus poderosas energías para que los agentes humanos puedan ejercitarse en la salvación de los perdidos". ${ }^{8}$

En la siguiente cita, Elena G. de White afirma que los grupos pequeños deben establecerse en cada iglesia y que estos deben tener una orientación misionera: 
"En cada iglesia debe haber grupos misioneros bien organizados para trabajar en el vecindario de esa iglesia. Poned el yo detrás de vosotros y dejad que Cristo vaya delante como vuestra vida y poder". 9

También Elena G. de White dejó valiosos consejos relativos al contenido de las reuniones en los grupos pequeños. Sobre el estudio de la Biblia y la oración en las reuniones, ella escribió:

"Reúnanse pequeños grupos por las tardes, al mediodía, o temprano en la mañana para estudiar la Biblia. Tengan un momento de oración, para que el Espíritu Santo los fortalezca, ilumine y santifique." 10

También ella consideró que una parte esencial de la reunión en el grupo pequeño, es el momento cuando los creyen-

${ }^{9}$ White, Ministerio de la Bondad, 112.

${ }^{10}$ White, Testimonios para la iglesia 7, 186. tes tienen la oportunidad de compartir su testimonio personal. En el contexto de una serie de orientaciones a quienes se reúnen en grupos pequeños, ella declaró:

"Qué hermoso testimonio podrán dar del amor manifestado entre compañeros de trabajo durante esos preciosos momentos de buscar la bendición de Dios. Que cada uno relate su propia experiencia con palabras sencillas. Esto traerá más consuelo y alegría al alma que todos los instrumentos de música que pudieran reunirse en las iglesias". ${ }^{11}$

Elena de White también recomienda que se promueva la más amplia participación posible en las reuniones del grupo pequeño:

"Que la alabanza a Dios salga de vuestros labios cuando os reunáis en los pequeños grupos

${ }^{11}$ White, Testimonios para la iglesia 7, 186. 
para adorar a Dios. Que no sea un solo hombre el que hable todo el tiempo. Permitid que varios participen". ${ }^{12}$

Estas y otras citas enfatizan la importancia de organizar grupos pequeños en la iglesia adventista. Por otro lado, ella también resaltó cuáles serían los resultados que se obtendrían al organizarnos conforme a la orientación divina. Escribiendo acerca de los grupos misioneros bien organizados, ella afirmó:

"Cuando tales fuerzas comiencen a trabajar en todas nuestras iglesias, habrá un poder renovador, reformador $y$ vigorizante, una reforma de enérgico poder en las iglesias, porque los miembros estarán haciendo la verdadera obra que Dios les ha dado para realizar". ${ }^{13}$

Sin duda, esta última cita es llamativa, porque Elena G.

${ }^{12}$ White, El ministerio pastoral, 303.

${ }^{13}$ Elena de White, El ministerio de la bondad, 112. de White nos reconviene en el sentido que si no estamos trabajando sobre la base de los grupos bien organizados, no estamos haciendo la "verdadera obra" que Dios nos ha dado para realizar.

\section{Promueve el crecimiento espiritual de la Iglesia}

Otra razón para el establecimiento urgente de los grupos pequeños en la iglesia, es que estos promueven el crecimiento espiritual de la iglesia. Si bien, el crecimiento espiritual no es algo que pueda fácilmente ser medido, es claro que un clima de excitación y una atmósfera emocionalista no son el termómetro adecuado para medir lo que llamamos crecimiento espiritual. Fundados en la Palabra de Dios, podemos mencionar algunos de los más importantes componentes del crecimiento espiritual, entre ellos el estudio de la Palabra de Dios. La Biblia nos dice: "Así que la fe es por el oír, y el oír, por la palabra de Dios" (Romanos 10:17). Por 
lo que concluimos que cuanto más se estudie y practique las enseñanzas de la Biblia, más aumentará nuestra fe.

Otro aspecto que influye en el crecimiento espiritual, es la relación de amor y amistad entre los creyentes. Jesús declaró: "En esto conocerán todos que sois mis discípulos, si os tenéis amor los unos a los otros" (Juan 13:35). Es decir, las relaciones caracterizadas por un verdadero amor práctico, identifican a los auténticos discípulos de Cristo. La iglesia

primitiva nos muestra en forma vívida este principio. Entre otras cosas, Lucas describe que ellos compartían "el pan en los hogares, comían juntos con alegría y sencillez de corazón" (Hechos 2:46).

Santiago sugiere que la oración y la alabanza constituyen también un aspecto que ayuda al crecimiento integral del cristiano (Santiago 5:13), mientras que el apóstol Pablo nos recuerda que trabajando por la salvación de otras personas, también nos mantenemos participando del gozo de la salvación (1 Timoteo 4:16).

Esos elementos: el estudio de la Biblia, la amistad cristiana, la oración y la alabanza, y el cumplimiento de la misión; son componentes vitales del crecimiento en la experiencia cristiana, y todos ellos se hayan presentes en las reuniones de los grupos pequeños. El plan de grupos pequeños es el que mejor provee la atmósfera ideal para el cultivo de esas cualidades espirituales por parte de cada uno de los miembros de la iglesia.

\section{Es una herramienta poderosa para la movilización de la iglesia}

Otro aspecto importante de los grupos pequeños es cómo pueden ser usados como una poderosa herramienta de movilización de la iglesia. Una iglesia que está organizada en pequeños grupos llega a funcionar como un disciplinado ejército. Por supuesto, hay un periodo de maduración que va desde la implantación de los grupos 
pequeños hasta su uso como herramienta de movilización misionera. Sin embargo, una vez que se ha consolidado el funcionamiento de los grupos y se ha inspirado a los líderes para ser verdaderos pastores de su pequeño rebaño, la movilización misionera suele ser masiva y llega a envolver a casi la totalidad de la iglesia.

Cuando la iglesia está organizada en grupos pequeños puede emprender casi cualquier proyecto misionero con la seguridad de obtener una respuesta masiva de los miembros. Por ejemplo, cuando los niveles superiores de la iglesia promueven la distribución masiva de publicaciones, no es necesario que los líderes del Ministerio Personal organicen el trabajo para toda la iglesia, solo necesitan convocar a los líderes de los grupos pequeños, darles las indicaciones y los materiales, y estos podrán organizar el trabajo entre sus miembros. Otro ejemplo, si el plan de evangelismo incluye el establecimiento de centros de predicación, el líder de Ministerio Personal de la iglesia puede desafiar a cada grupo pequeño que nombre su predicador y organice sus comisiones para tener su propio centro de predicación. Si se trata de una semana de cosecha en el templo, cada grupo puede organizarse para visitar a sus invitados y llevarlos a las reuniones del templo. Si se trata de una semana especial a través de la TV, cada grupo puede conseguir la logística necesaria y adecuar el lugar a la programación de la semana. Aun si se trata de almuerzos de iglesia, salidas, etc. Todo puede ser realizado a través de los grupos pequeños. ${ }^{14}$ Lo interesante del caso es que no es necesario crear una

\footnotetext{
${ }^{14}$ Que todo se puede hacer a través de los grupos pequeños, no significa necesariamente que todo se deba hacer a través de los grupos pequeños. Las actividades que se hagan en los grupos pequeños dependerán del énfasis que la iglesia coloque sobre ellos. Es evidente que la estructura de grupos pequeños puede ser usada de manera inconveniente, pero eso sería un defecto en el empleo de los grupos pequeños, no en la naturaleza misma de ellos.
} 
estructura para cada programa porque los grupos pequeños son una estructura permanente y que está siempre dispuesta a las demandas de las actividades eclesiásticas, especialmente a aquellas relativas al cumplimiento de la misión.

\section{Promueve el evangelismo como estilo de vida en la iglesia}

Una razón muy importante para la implementación de los grupos pequeños en la iglesia es que a través de ellos se promueve el evangelismo como un estilo de vida de la iglesia. Una iglesia que trabaja con base en los grupos pequeños se encuentra haciendo evangelismo durante todos los días del año. No hay una fecha específica en el calendario donde se inicia la campaña de evangelismo pues la iglesia realiza su labor en favor de los perdidos de manera permanente. Recordando a Elena G. de White, en relación al trabajo en grupos pequeños ella dice: "Únanse diferentes personas en el trabajo como pescadores de hombres. Traten de recoger a las almas de la corrupción del mundo y conducirlas a la pureza salvadora del amor de Cristo". ${ }^{15}$ Una estrategia adecuada para la obra misionera es establecer, siguiendo el ejemplo de Jesús, las parejas misioneras en el seno del grupo pequeño. A través de las parejas misioneras se pueden impartir los estudios bíblicos y el conocimiento doctrinal básico, en tanto que en el grupo pequeño se proporcionan la atmósfera de aceptación y amor cristiano que permitirá el crecimiento espiritual de los estudiantes de la Biblia. No hay pues ninguna incompatibilidad entre los grupos pequeños y las parejas misioneras, por el contrario, toda estrategia de evangelismo debe trabajar con estos métodos de un modo armonioso y totalmente complementario.

Un aspecto importante es que el evangelismo a través de los grupos pequeños no es rea-

${ }^{15}$ White, Evangelismo, 88. 
lizado de modo elitista por un cuerpo especializado de obreros bíblicos mientras la gran mayoría de miembros no manifiesta el menor compromiso. En el plan de grupos pequeños todos pueden participar del evangelismo a través del uso de sus propios dones espirituales. En un grupo pequeño bien organizado, cada miembro siente la responsabilidad de traer continuamente invitados a la reunión. Cuando asiste un invitado, cada miembro tiene un grado de influencia en la constitución de una atmósfera adecuada para que esa persona llegue a los pies de Jesús. Una pareja de grupo misionero suele tomar la responsabilidad de impartir estudios bíblicos. Los miembros del grupo visitan y animan al recién convertido. Cuando una persona toma la decisión de bautizarse, todos en el grupo han colaborado de alguna manera en esa victoria espiritual. ${ }^{16}$

\footnotetext{
${ }^{16}$ En la experiencia del autor, los gru-
}

Es un excelente método para la consolidación y el crecimiento espiritual de los nuevos creyentes.

El hecho de que el grupo pequeño ayude decisivamente en la consolidación y el crecimiento espiritual de los nuevos creyentes, es una razón para implementar los grupos pequeños en la iglesia. En el contexto de los grupos pequeños, se produce la ganancia de almas por el trabajo con los no convertidos, pero realizar el trabajo misionero es el ingrediente fundamental para mantener viva la

pos pequeños han sido el soporte ideal para la formación y consolidación de las parejas misioneras. Una pareja misionera puede ser el núcleo para iniciar un grupo pequeño, y a medida que el grupo se consolida se va promoviendo la aparición de nuevas parejas misioneras entre los miembros y los recién convertidos. Por otro lado, el énfasis de la pareja misionera es la visitación en las casas y los estudios bíblicos secuenciales, en tanto que el grupo pequeño se centra en la amistad y el compañerismo cristiano. Ambos aspectos son esenciales para el crecimiento espiritual de un recién convertido. $\mathrm{Y}$ no son, en ningún modo, incompatibles. 
fe del creyente, por lo que se consolida su experiencia cristiana. Es decir, se configura un escenario en que el evangelismo y la consolidación son las dos caras de una misma moneda. No es posible separar ambos procesos, porque el recién convertido puede empezar a participar del evangelismo al invitar a sus allegados al grupo pequeño, y al evangelizar y participar de las reuniones, está consolidando su fe.

Cuando en el grupo pequeño, una persona acepta a Cristo y es bautizado, tiene a su lado un grupo de apoyo que será crucial en su crecimiento espiritual. El grupo pequeño no termina su tarea al llevar a una persona al bautismo, simplemente continúa su obra de soporte espiritual. El nuevo converso no se siente solo, porque su grupo pequeño continúa reuniéndose, y en las reuniones se continúa orando y estudiando la Biblia con él. Fuera de las reuniones, los miembros del grupo pequeño continúan visitándolo. De esta manera, cual recién naci- do, el nuevo creyente se siente amparado y acompañado en los primeros pasos de su caminata con Cristo.

La conservación o confirmación es un elemento básico para el crecimiento de la iglesia. Un axioma indiscutible de crecimiento de iglesia es que sin conservación no hay crecimiento. En este contexto, no es sostenible decir que se está bien en relación con el crecimiento de la iglesia mientras que la puerta de atrás se mantiene abierta de par en par. Es claro que si no se está bien en la conservación, no se estará bien en el crecimiento de la iglesia. El plan de grupos pequeños no se interesa apenas por llevar una persona hacia la decisión del bautismo, sino que la sigue ayudando en su crecimiento cristiano hasta convertirla en un verdadero discípulo de Jesús.

Promueve el surgimiento de nuevos líderes en la iglesia

Muchas personas que no se atreven a liderar en la iglesia inician sus primeros pasos como líderes de iglesia en su pe- 
queño grupo. El grupo pequeño es un extraordinario semillero de nuevos líderes. Uno de los pilares de la continuidad y el fortalecimiento de los grupos pequeños, es la disponibilidad de nuevos líderes en la iglesia. Esos líderes no surgen de modo espontáneo, es necesario tener un plan para producir el surgimiento de nuevos líderes.

Una característica básica de un líder es que tiene seguidores. Si una persona no tiene seguidores, no puede considerarse un líder. Muchas veces llamamos "líder" a una persona que ocupa algún cargo en la iglesia. Sin embargo, no todo oficial de iglesia es un líder, y muchas veces hay personas que no fueron nombradas para cargo alguno en la iglesia, y sin embargo son líderes por el grado de influencia que tiene sobre las personas. En el contexto de una iglesia organizada en grupos pequeños, los líderes tienen seguidores que son los miembros de su grupo pequeño. Por lo que la gran prioridad es motivar, entrenar y equipar al líder para que pueda ejercer su liderazgo de la mejor forma posible. Eso hace indispensable un programa continuo de capacitación de líderes. Esta labor es tan importante que podríamos decir que el plan de grupos pequeños no tiene como meta principal formar continuamente nuevos grupos, sino capacitar permanentemente a los líderes y promover activamente el surgimiento de nuevos líderes, porque en la medida en que más líderes sean enviados a ministrar, más grupos pequeños surgirán.

\section{Es un programa que maximiza los recursos financieros de la iglesia}

El plan de grupos pequeños demanda una ingente cantidad de recursos humanos, y una gran dosis de sacrificio personal. Se necesitan líderes que estén dispuestos a darse a sí mismos por la causa del evangelio. Sin embargo, no es necesario disponer de una gran cantidad de recursos fi- 
nancieros. Con el debido entrenamiento de los líderes y de la iglesia, se puede alcanzar a muchas personas para Cristo. Se necesitan Biblias, lecciones bíblicas y programas, no mucho más. Por otro lado, los recursos pueden ser canalizados para los emprendimientos evangelísticos en lugares donde no hay presencia adventista y en las reuniones públicas. El plan de grupos puede ser realizado con un costo significativamente menor al de cualquier otro método de evangelismo.

\section{Ha sido aplicado con éxito en los más variados contextos}

Es interesante cómo los grupos pequeños se han desarrollado en los más variados contextos socio-económicos. Hay historias de gran éxito desde los diferentes países que componen nuestra DSA. Han surgido grupos pequeños en las iglesias de la ciudad y en las iglesias de las regiones rurales. Se han implementado los gru- pos en iglesias pequeñas y en iglesias grandes. ${ }^{17}$

Los principios de aplicación de los grupos pequeños deben contextualizarse en cada escenario. En algunos lugares, la implementación llevará mucho más tiempo, pero si se toman las debidas previsiones y se tiene una adecuada estrategia de implementación, los grupos pequeños podrán finalmente ser implantados.

En las iglesias tradicionales, y de gran número de miembros, el desafío será mucho mayor que iglesias más pequeñas. Sin embargo, como fue mencionado anteriormente, Elena G. de White declaró: "En cada iglesia debe haber grupos misioneros

${ }^{17}$ En la propia experiencia ministerial del autor, se ha establecido grupos pequeños en iglesias de treinta o cuarenta miembros (En las ciudades de Tumbes y Teófilo Otoni y Nanuque en Minas Gerais, Brasil), en iglesias de tamaño medio (Havaí en Belo Horizonte, y Santa Isabel en Lima, Perú). Una última experiencia estamos implementando actualmente en la Iglesia de Villa Unión, de más de mil miembros, donde se han organizado ciento veinte grupos pequeños. 
bien organizados para trabajar en el vecindario de esa iglesia" ${ }^{18}$, y no podemos pensar que Dios nos haya pedido que se organicen en cada iglesia si fuese imposible hacerlo en alguna. Como líderes debemos someternos a la orden del Señor y dejarnos guiar por su mano, pidiéndole sabiduría para implementar su plan divino en el contexto específico de nuestra iglesia local.

\section{Promueve el desarrollo de los dones del pastor y de la iglesia}

Finalmente, otro aspecto importante para el establecimiento de los grupos pequeños, es la manera en que este plan ayuda a desarrollar las habilidades y los dones del pastor, los líderes, y la iglesia en general.

En el plan de grupos pequeños, el liderazgo pastoral tiene un rol preponderante. Solo el

${ }^{18}$ White, Ministerio de la bondad, 112. pastor tiene la llave del éxito del plan en su distrito. ${ }^{19}$ Hay oportunidades ilimitadas para que el pastor pueda desenvolver sus habilidades particulares: en la visitación, en la organización de eventos, en la estrategia misionera, en la predicación, en la movilización misionera, en el liderazgo, en la administración, etc. Del mismo modo, los líderes y los miembros de la iglesia tienen un amplio campo de acción para el uso y el desarrollo de sus habilidades y talentos.

Alguien podría preguntarse ¿Cuál es el rol del pastor y de los líderes de iglesia si todo lo hacen los grupos pequeños? Evidentemente, los grupos no actúan de modo espontáneo sino que lo hacen porque hay líderes que los motivan y los

${ }^{19} \mathrm{El}$ trabajo del pastor es clave en la capacitación continua de los líderes de los grupos pequeños, la implementación decidida del plan y la supervisión permanente de los grupos pequeños. La iglesia le dará énfasis al plan de grupos pequeños solo si percibe que la formación de grupos pequeños es la base del esfuerzo cristiano en el ministerio de su pastor. 
orientan. En el plan de grupos pequeños, el pastor debe ejercer un liderazgo capacitador, motivador y visionario sobre sus líderes. En el caso de los oficiales de la iglesia, los grupos pueden convertirse en el soporte ideal para cumplir los objetivos de su departamento. El líder del Ministerio Personal, por ejemplo, puede contar con líderes de grupos pequeños que lo ayuden a alcanzar los objetivos misioneros de la iglesia, a cambio, debe motivarlos, entrenarlos,

62 equiparlos y evaluarlos para comprometerlos en la actividad misionera. Del mismo modo, otros departamentos pueden trabajar mejor a través de los grupos pequeños. ${ }^{20}$

${ }^{20}$ En varias de las iglesias del autor, la escuela sabática y las reuniones de jóvenes fueron muy fortalecidas cuando se promovió la participación de los grupos pequeños. También los grupos pequeños ayudaron en la mayoría de las iglesias a multiplicar el número de mayordomos fieles, en algunos casos, como el del distrito de Tumbes, a casi el 100\%.

\section{Conclusión}

Para finalizar, presentamos las siguientes conclusiones obtenidas a la luz del presente estudio:

La organización de los grupos pequeños en la iglesia para colocarlos como base del esfuerzo cristiano, es un plan que viene de Dios.

Todas las iglesias deberían contar con una sólida estructura de grupos pequeños para trabajar tanto por la iglesia como por los que no conocen a Jesús.

La capacitación permanente de los líderes de es un aspecto vital para el fortalecimiento y la consolidación de los grupos pequeños.

No hay incompatibilidad entre el trabajo de grupos pequeños y el de las parejas misioneras. Una pareja misionera puede liderar un grupo pequeño, y debe promover activamente el surgimiento de nuevas parejas misioneras en su grupo pequeño.

Implementar grupos pequeños en la iglesia es un desafío que no está exento de dificulta- 
des; hay muchos obstáculos que superar, hay muchas costumbres establecidas que deben ser superadas. Pero el Señor nos llama a avanzar con fe, sabiendo que quien nos encomendó esta tarea es "uno que no puede errar". 